\title{
NEW FRONTIERS IN NUCLEAR PHYSICS RESEARCH AT ELI-NP*
}

\author{
C.A. Ur, D. Balabanski, G. Cata-Danil, S. Gales, I. Morjan \\ O. Tesileanu, D. Ursescu, I. Ursu, N.V. Zamfir \\ Extreme Light Infrastructure-Nuclear Physics, IFIN-HH \\ 077125 Bucharest-Magurele, Romania
}

(Received February 2, 2015)

\begin{abstract}
Extreme Light Infrastructure-Nuclear Physics (ELI-NP) is one of the three pillars of the pan-European ELI initiative aiming to use extreme electromagnetic fields for nuclear physics research. The pillar, currently under construction at Bucharest-Magurele, will comprise two major research instruments: a high power laser system and a very brilliant gamma beam system. Both systems are at the limits of the present-day's technology. The high power laser system will consist of two 10 PW APPOLON-type lasers based on OPCPA technology with output energy higher than $200 \mathrm{~J}$, pulse duration of 20-30 fs and intensities of up to $10^{23}-10^{24} \mathrm{~W} / \mathrm{cm}^{2}$. The gamma beam, produced via inverse Compton scattering of laser pulses on a relativistic electron beam, will be characterized by high spectral density of about $10^{4}$ photons $/ \mathrm{s} / \mathrm{eV}$, narrow bandwidth $(<0.5 \%)$, tunable energy of up to $20 \mathrm{MeV}$ and degree of linear polarization higher than $95 \%$.
\end{abstract}

DOI:10.5506/APhysPolB.46.743

PACS numbers: 41.75.Jv, 07.85.Fv, 29.20.Ej, 25.20.-x

\section{Introduction}

In 2006, the European Strategy Forum on Research Infrastructure (ESFRI) listed the Extreme Light Infrastructure (ELI) [1] on its road-map [2] as a pan-European Research Infrastructure initiative. At the end of the preparatory phase (ELI-PP), it was decided to have a distributed infrastructure with three pillars implemented in different countries: (1) ELI Beamlines in Prague (Czech Republic) focused on the production of ultra intense and ultra short pulses of electrons, protons and ions; (2) ELI Attosecond in Szeged (Hungary) dedicated to the investigation of electron dynamics in

* Presented at the Zakopane Conference on Nuclear Physics "Extremes of the Nuclear Landscape", Zakopane, Poland, August 31-September 7, 2014. 
atoms, molecules, plasmas and solids at attosecond level; (3) ELI-Nuclear Physics in Bucharest (Romania) dedicated to laser-based nuclear physics research. The implementation of the project started in 2011 and the ELI-NP pillar is scheduled to enter operation in 2017.

Within the ELI-NP pillar two well-established scientific communities, high-power lasers and nuclear physics, have joined their efforts to build a new interdisciplinary facility and to define its research program. As a result of this collaboration, the scientific interest of ELI-NP is covering a broad range of key topics in frontier fundamental physics and new nuclear physics. The scientific case of ELI-NP was elaborated by an international collaboration of more than 100 scientists from 30 countries and published as the ELI-NP White Book [3]. The main research topics of interest are: (i) laserdriven nuclear physics experiments, (ii) characterization of the laser-target interaction by the means of nuclear physics instruments, (iii) photonuclear reactions, (iv) exotic nuclear physics and astrophysics. A particular attention is also given to the development of innovative applications based on the use of both high-power lasers and brilliant, narrow bandwidth gamma beams.

This paper gives an overview of the ELI-NP facility, the status of its implementation and the perspectives for nuclear physics research.

\section{The ELI-NP research facility}

The research facility ELI-NP is being currently under construction on the Magurele Physics Platform in southern Bucharest. The new facility will occupy a total surface of about $33,000 \mathrm{~m}^{2}$. The main building, covering nearly half of this area, is dedicated to the experimental activities and it will host: (i) the main research equipment, (ii) experimental rooms, (iii) laboratories and workshops, (iv) control rooms and user area. Other buildings under construction in the same area are the office building, a guest house and a canteen. A schematic view of the experimental building is shown in Fig. 1.

Civil engineering construction has started in June 2013 and should be completed by the summer of 2015 . Figure 2 shows the construction status as of August 2014.

The major equipment hosted by ELI-NP will provide to the users laser and gamma beams with unprecedented parameters allowing the facility to cover frontier fundamental physics, new nuclear physics and astrophysics as well as applications in material and life sciences, industrial tomography, nuclear waste management. 


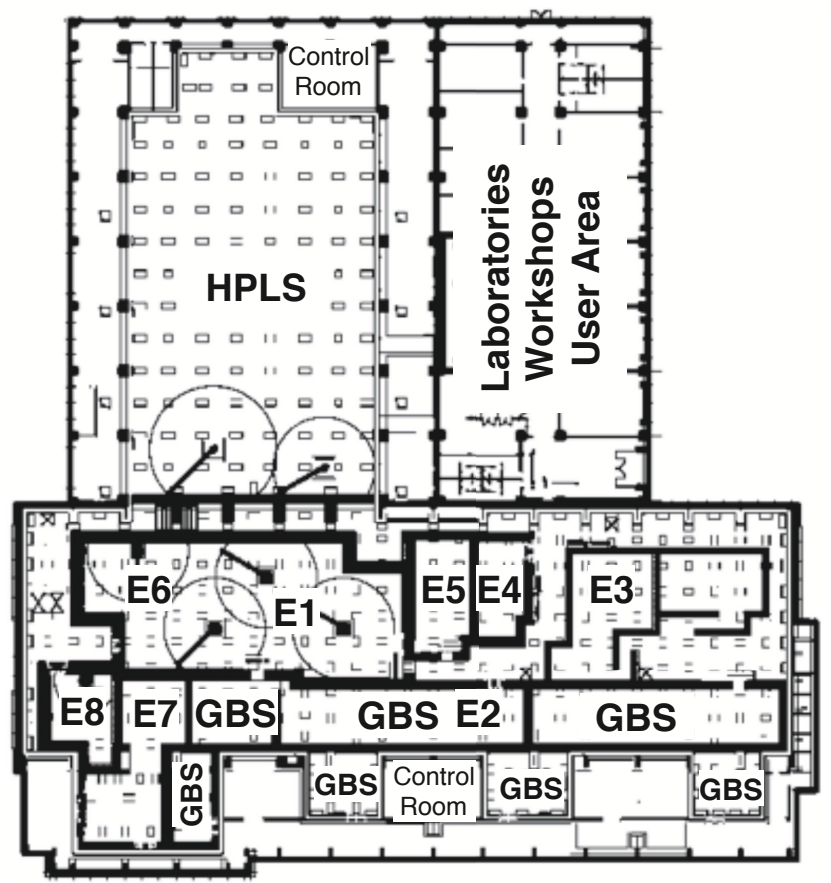

Fig. 1. A schematic layout of the ELI-NP experimental building showing the main areas for the implementation of the research equipment and support laboratories. The meaning of the labels is as follows: HPLS - high-power laser system, GBS gamma beam system, E1... E8 - experimental rooms.

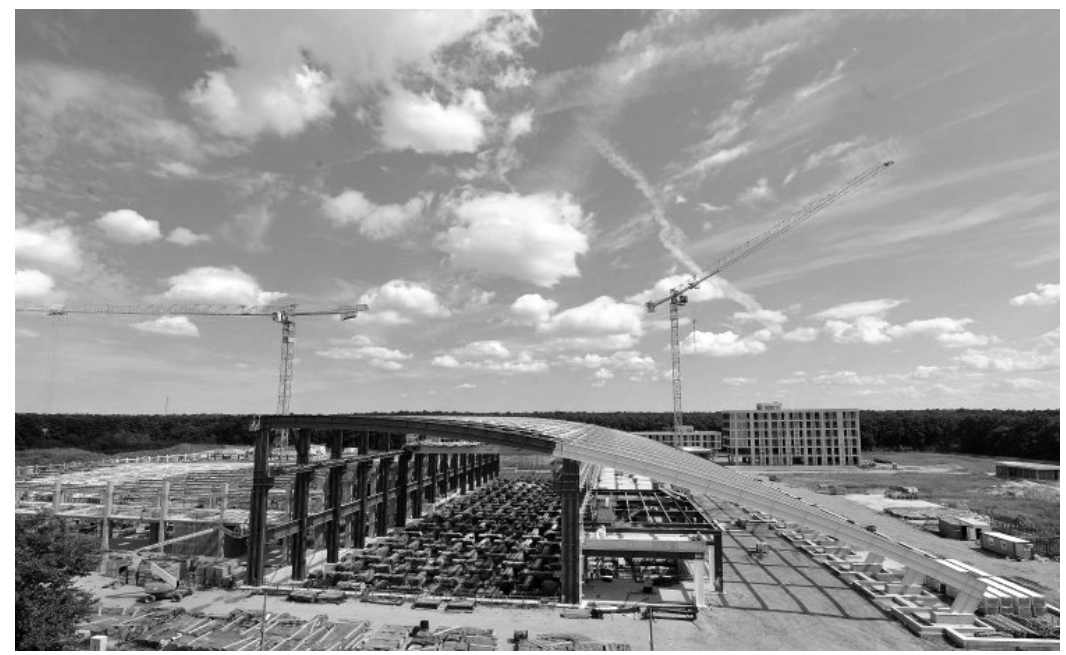

Fig. 2. Image of the ELI-NP building site taken as of August 2014. The experimental building and the office building are visible. 


\subsection{The high-power laser system}

The high-power laser system (HPLS) of ELI-NP consists of two 10 PW class lasers based on Optical Parametric Chirped Pulse Amplification (OPCPA) $[4,5]$ and driven by a dual front-end system with two parallel amplification arms. Each amplification arm will have three outputs each with its own optical pulse compressor. The three outputs will provide different power levels. Besides the $10 \mathrm{PW}$ output at a repetition rate of 1/60 $\mathrm{Hz}$ there will be two other outputs of $100 \mathrm{TW}$ and $1 \mathrm{PW}$ at repetition rates of $10 \mathrm{~Hz}$ and $1 \mathrm{~Hz}$, respectively.

Out of the six possible outputs on the two arms, two of them, one from each arm, can be provided simultaneously for experiments, combined in the same experimental setup or to be used independently in two different setups. For the two 10 PW outputs an unprecedented level of intensity of about $10^{23}$ $10^{24} \mathrm{~W} / \mathrm{cm}^{2}$ will be achieved. The HPLS is being built by Thales Optronique France and Thales Romania. The main parameters characterizing the ELINP HPLS are summarized in Table I.

TABLE I

Main parameters of the ELI-NP high-power laser system [6].

\begin{tabular}{ll}
\hline \hline \multicolumn{1}{c}{ Parameter } & \multicolumn{1}{c}{ Value } \\
\hline Outputs: & 2 outputs: $10 \mathrm{PW} @ 1$ shot $/ \mathrm{min}$ \\
power and & 2 outputs: $1 \mathrm{PW} @ 1$ shot/s \\
repetition rate & 2 outputs: $0.1 \mathrm{PW} @ 10$ shot/s \\
Pulse duration & $<50 \mathrm{fs}$ \\
Strehl ratio & $>0.7$ \\
Pointing stability & $<0.2 \mu \mathrm{rad}$ \\
Temporal contrast in & $10^{11}: 1$ for the $0.1 \mathrm{PW}$ outputs \\
the nanosecond range & $10^{12}: 1$ extrapolated for the $10 \mathrm{PW}$ outputs \\
Temporal contrast in & $10^{11}: 1$ for the $0.1 \mathrm{PW}$ outputs \\
the picosecond range & $10^{12}: 1$ extrapolated for the $10 \mathrm{PW}$ outputs \\
External clock sync. jitter & $<200 \mathrm{fs}$
\end{tabular}

\subsection{The Gamma Beam System}

The Gamma Beam System (GBS) for ELI-NP was designed to provide a very intense and brilliant gamma beam with tunable energy based on the incoherent inverse Compton scattering of a high repetition pulsed laser light off a high intensity, low emittance, relativistic electron beam with energies up to $720 \mathrm{MeV}$ [7]. The experiments proposed to be performed with the gamma beam at ELI-NP [3] have imposed the following key parameters of 
the gamma beam: bandwidth (bwd) better than $0.5 \%$ with spectral densities of 10,000 photons $/ \mathrm{s} / \mathrm{eV}$, photons energy in the range $0.2-19.5 \mathrm{MeV}$, peak brilliance higher than $10^{21}$ photons $/ \mathrm{mm}^{2} / \mathrm{mrad}^{2} / \mathrm{s} /(0.1 \% \mathrm{BW})$, high degree of linear polarization (higher than 95\%).

The building and installation of the ELI-NP GBS will be performed by EuroGammaS [7], a European Consortium of academic and research institutions and industrial partners with expertise in the field of electron accelerators and laser technology. The main components of the GBS are: the electron accelerator, the interaction lasers, the laser recirculators and the collimation systems.

The electron accelerator is a high brightness normal conducting linac consisting of two S-band and twelve C-band RF structures. The main advantage of using a linac accelerator is the excellent emittance of the provided electron beams. The accelerator will be operated at a radio-frequency (RF) repetition rate of $100 \mathrm{~Hz}$. For every RF pulse 32 electron micro-bunches, separated at $16 \mathrm{~ns}$, will be accelerated. Every micro-bunch will have an electrical charge of $250 \mathrm{pC}$. The time structure of the electron beam is shown in Fig. 3. The accelerator is designed in two stages: the first stage will produce electrons with energies up to $300 \mathrm{MeV}$, while in the second stage the electrons will be further accelerated up to $720 \mathrm{MeV}$.

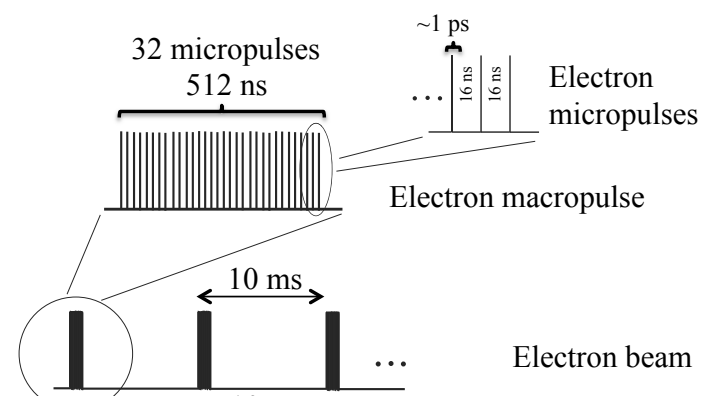

Fig. 3. Time structure of the electron beam provided by the ELI-NP normal conducting linac. The RF repetition rate is of $100 \mathrm{~Hz}$. At every RF pulse, a train of 32 electron micro-bunches, with $250 \mathrm{pC}$ charge and about 1 ps time duration each, is generated. The time separation between the micro-bunches is of 16 ns.

The two interaction lasers are cryo-cooled high average power, high quality J-Class diode pumped Yb:YAG lasers. They will operate at $100 \mathrm{~Hz}$ repetition rate and they will deliver green light $(515 \mathrm{~nm})$ with energy of $0.2 \mathrm{~J}$. The energy bandwidth ( $\mathrm{rms}$ ) is of $0.1 \%$ and pointing stability of the laser light is of $1 \mu \mathrm{rad}$. One of the lasers will be used for low-energy (up to $3.5 \mathrm{MeV}$ ) gamma beams production and both of them are used for the production of high-energy (up to $19.5 \mathrm{MeV}$ ) gamma beams. 
Laser recirculation is needed to match the electron beam time structure (see Fig. 3) with the $100 \mathrm{~Hz}$ repetition rate of the lasers. To ensure the interaction of every laser pulse with the corresponding 32 electron microbunches, the laser light will be inserted in an optical recirculator [8] with multi-pass geometry and a fixed focus point. The recirculator has to ensure that the collision between the laser light and the electron micro-bunches occurs always at the same incident angle and that the polarization of the gamma beam is preserved. The synchronization of the electron accelerator $\mathrm{RF}$ with the interaction lasers front-end is done to better than $0.5 \mathrm{ps}$.

To achieve the target bandwidth of better than $0.5 \%$ for the gamma beam produced following the inverse Compton scattering of the laser pulse off the electron beam, one has to take advantage of the strong angle-energy correlation of the scattered photons. By placing a collimator in the scattered photon beam, one can select the energy and the desired bandwidth by continuously adjusting the collimator aperture. The main requirements for the design of the gamma beam collimators are: low transmission of the gamma-ray photons, continuously adjustable aperture and minimization of the primary beam contamination with production of secondary radiation.

The parameters of the ELI-NP GBS are summarized in Table II.

TABLE II

Main parameters of the ELI-NP Gamma Beam System [7].

\begin{tabular}{ll}
\hline \hline \multicolumn{1}{c}{ Parameter } & \multicolumn{1}{c}{ Value } \\
\hline Energy [MeV] & $0.2-19.5$ \\
Spectral density $[\mathrm{ph} / \mathrm{s} / \mathrm{eV}]$ & $10^{4}$ \\
Bandwidth rms [\%] & $<0.5$ \\
$\#$ Photons/shot within FWHM bdw. & $\leq 8.3 \times 10^{8}$ \\
Source rms size $[\mu \mathrm{m}]$ & $10-30$ \\
Source rms divergence $[\mu \mathrm{rad}]$ & $25-200$ \\
Peak brilliance [Nph/s mm ${ }^{2} \mathrm{mrad}^{2} 0.1 \%$ bdw] & $10^{20-10^{23}}$ \\
Pulse length rms [ps] & $0.7-1.5$ \\
Linear polarization [Macro repetition rate [Hz]] & 100 \\
Number of pulses/macropulse & 32 \\
Pulse-to-pulse separation [ps] & 16
\end{tabular}




\section{The scientific program}

The scientific program of ELI-NP defined in the White Book [3] is based on the unique features of the high-power laser and gamma beams as discussed in the previous sections.

A block diagram of the ELI-NP infrastructure is given in Fig. 4 showing schematically the arrangement of the beams, their possible combinations and delivery in the experimental rooms. The experimental activity of the facility can be divided in three main categories: (1) high-power laser studies in E1, E4, E5 and E6; (2) experiments with gamma beams in E2, E3 and E8; (3) combined measurements with high-power lasers and gamma beams in E7.

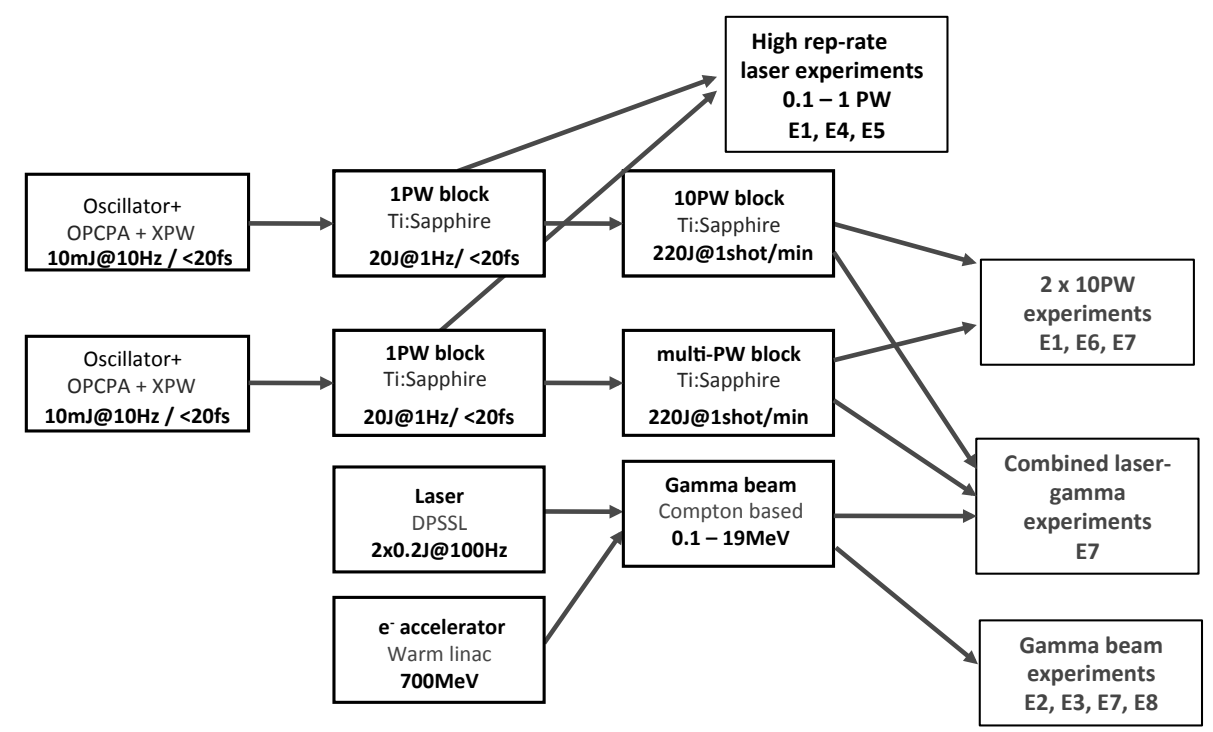

Fig. 4. Block diagram of the ELI-NP facility.

There are several areas where the experimental setups will be installed. Depending on the type of beams that can reach the areas their destination of use is as follows:

- E1 is dedicated to laser-driven nuclear physics experiments. Laser acceleration of heavy ions will be investigated and the possibility to use it for nuclear reactions. Acceleration of ions with high-power lasers offers unique features such as production of beams with solid target densities and large acceleration over very short distances. The flagship experiment is aiming at the study of neutron-rich nuclei in the region of $N=126$ r-process waiting point by using acceleration of Th ions and their subsequent fission [9]. 
- E2 will be used for Nuclear Resonance Fluorescence (NRF) experiments with low-energy (up to $3.5 \mathrm{MeV}$ ) gamma beams. The high brilliance and small diameter of the beam will increase the sensitivity of the measurements and will require less material (of the order of few $\mathrm{mg}$ ) for the construction of the targets. This opens the possibility to study nuclei with limited availability such as the $p$-nuclei or with large radiation background such as actinides. The NRF technique allows the recovery of several physical quantities in a model independent way, such as: the excitation energies, level widths $\Gamma$, decay branching ratios or spin quantum numbers and parities of the excited nuclear states. Applications of the NRF method to characterize the content of samples in given isotopes are also considered; the tunable gamma beam energy at ELI-NP will allow for specific isotopes identification based on NRF while the location in the sample can be made based on gamma-ray computerized tomography. Highly efficient and accurate nuclear waste content characterization for determination of the ${ }^{235} \mathrm{U} /{ }^{238} \mathrm{U}$ ratio in spent fuel rods or determination of ${ }^{239} \mathrm{Pu}$ content will become possible.

- E3 is planned to be used for material physics studies with positrons. Positrons will be produced by the gamma beams in a converter foil via the $\left(\gamma, e^{-}-e^{+}\right)$process in the first accelerator bay. After moderation, the positrons will be transported in the E3 room for experiments. Moderated positrons are useful for material science as probes for defect spectroscopy. One can investigate the type and concentration of defects to depths up to several $100 \mathrm{~nm}$.

- E4/E5 are the high repetition areas where laser beams with powers of $0.1 \mathrm{PW}$ and $1 \mathrm{PW}$ will be available. One can take advantage of the laser driven radiation production, such as ultra-short time scale and the relatively broadband spectrum secondary radiation, to understand the material behavior under extreme conditions of radiation.

- E6 will host experiments related to strong-field quantum electrodynamics. High intensity laser pulses of about $10^{23} \mathrm{~W} / \mathrm{cm}^{2}$ will be sent on solid targets. Electron-positron production in laser-irradiated solid targets and their subsequent behavior will be the main object of study [10].

- E7 is dedicated to combined experiments with high-power lasers and gamma beams. The possibility to study the same target with these very different brilliant beams will be unique and shall reveal new phenomena. Laser and gamma radiations can be used in this case as pump and probe, in order to excite processes and observe the subsequent evolution of the systems. 
- E8 is the area dedicated to experiments with gamma beams of energy up to $19.5 \mathrm{MeV}$. Pygmy Dipole Resonances (PDR) can be investigated above and below the particle threshold, which is essential for nucleo-synthesis in astrophysics. PDRs occur close to the neutron emission threshold and their decay is governed by the coupling to the large number of states around the threshold. The use of ELINP gamma beam provides the possibility to reveal possible fine structures/splitting of PDRs the excitation function with high resolution for $(\gamma, n)$ and $(\gamma$, charged particle) channels, allowing for the determination of the branching ratios for various decay channels. The linear polarization of the beam will allow for the determination of the E1 or M1 type of excitation for the observed structures.

The ELI-NP facility provides unique opportunities for nuclear astrophysics research. Gamma-induced nuclear reactions of astrophysical interest still represent a challenge due to their very low cross sections as the reactions occur deep below the Coulomb barrier. The study of these reactions will benefit from the use of the high intensity gamma beams at ELI-NP.

High-resolution photo-fission studies in the actinides, investigation of the second and third potential minima, angular and mass distribution measurements of fission fragments, measurements of absolute photo-fission cross sections, studies of rare photo-fission events, such as triple fission, highly asymmetric fission, etc. are considered for investigation with the gamma beams at ELI-NP.

A variety of applied research with high-energy gamma beams are also considered. New production schemes of radioisotopes for medicine by following $(\gamma, n)$ reactions are examined. The ${ }^{195 m} \mathrm{Pt}$ isotopes, used for nuclear imaging to determine the efficiency of chemotherapy, is one of the candidates. Computerized tomography with gamma-ray beams for non-destructive inspection of objects, will benefit from high-energy quasi-monochromatic and high beam intensity to shorten the scanning time.

\section{Conclusions}

ELI-NP is a new research infrastructure, part of the pan-European Extreme Light Infrastructure initiative, dedicated to nuclear physics driven by an ultra high-power laser system composed of two 10 PW lasers and by a brilliant, high intensity gamma beam system. The unique features of the two systems, well beyond the present-day state-of-the-art, open new opportunities for both fundamental nuclear physics and applied research. The definition of the physics cases and of the experimental setups is done within a broad international collaboration with universities and research institutions from all over the world. 
The ELI-NP project is co-funded by the European Union through the European Regional Development Fund.

\section{REFERENCES}

[1] ELI-Extreme Light Infrastructure Science and Technology with Ultra-Intense Lasers Whitebook, Editors G.A. Mourou, G. Korn, W. Sandner, J.L. Collier, at THOSS Media GmbH, 2011.

[2] European Strategy Forum on Research Infrastructures, European Roadmap for Research Infrastructures, Report 2006.

[3] ELI-NP White Book, http://www.eli-np.ro/documents/ELI-NP-WhiteBook.pdf

[4] D. Strickland, G. Mourou, Opt. Commun. 56, 219 (1985).

[5] I.N. Ross et al., Opt. Commun. 144, 125 (1997).

[6] D. Ursescu et al., High-Power, High-Energy, and High-Intensity Laser Technology; and Research Using Extreme Light: Entering New Frontiers with Petawatt-Class Lasers, edited by J. Hein, G. Korn, L. Oliveira Silva, Proc. of SPIE, Vol. 8780, 2013, p. $87801 \mathrm{H}$.

[7] O. Adriani et al., arXiv:1407.3669 [physics.acc-ph].

[8] K. Dupraz et al., Phys. Rev. ST Accel. Beams 17, 033501 (2014).

[9] D. Habs et al., Appl. Phys. B103, 471 (2011).

[10] R. Hajima et al., J. Nucl. Sci. Tech. 45, 441 (2008). 